\title{
Laser balloon ablation in patients with a left common pulmonary vein.
}

Shinichi Tachibana ${ }^{1}$, Kaoru Okishige ${ }^{2}$, Koji Sudo $^{1}$, Takatoshi Shigeta ${ }^{3}$, Yuichiro Sagawa ${ }^{4}$, Manabu Kurabayashi ${ }^{2}$, Yasuteru Yamauchi ${ }^{5}$, Masahiko Goya $^{6}$, and Tetsuo Sasano ${ }^{7}$

${ }^{1}$ Yokohama City Minato Red Cross Hospital

${ }^{2}$ Yokohama-city Port Red Cross Hospital

${ }^{3}$ Yokohama Bay Red Cross Hospital

${ }^{4}$ Yokosuka Kyousai Hospital

${ }^{5}$ Yokohama-city Bay Red Cross Hospital

${ }^{6}$ Tokyo Medical and Dental University Hospital

${ }^{7}$ Tokyo Medical and Dental University

October 31, 2021

\begin{abstract}
Background: Pulmonary vein isolation (PVI) with a balloon-based visually guided laser ablation (VGLA) is regarded as a useful therapeutic tool for treating atrial fibrillation (AF). The clinical efficacy of a VGLA has never been fully investigated in patients with a left common pulmonary vein (LCPV). Objective: We investigated the procedural safety as well as clinical usefulness of VGLA in patients with an LCPV. Methods: This study consisted of 130 consecutive patients who underwent VGLA of de novo non-valvular paroxysmal AF. Results: Eleven patients (8.5\%) had an LCPV (ostium maximal average diameter: $27.5 \pm$ $4.9 \mathrm{~mm}$, ostium minimal average diameter: $17.7 \pm 3.5 \mathrm{~mm})$. Nine out of $11(81.8 \%)$ LCPVs were successfully occluded and isolated at the ostium with a VGLA guided PVI. The ablation procedure time was significantly shorter in the patients with than without an LCPV $(61.5 \pm 15.4$ vs. $86.9 \pm 32.9 \mathrm{~min}, \mathrm{p}=0.01)$. There was no difference regarding the atrial tachyarrhythmia recurrence between those with and without an LCPV $(\mathrm{p}=0.18)$. A total of fifteen patients underwent a redo procedure, but reconnections were not observed in any of the LCPV patients. Conclusion: The VGLA guided PVI was a useful therapeutic tool even in patients with an LCPV. The presence of an LCPV was not associated with any atrial tachyarrhythmia recurrence.
\end{abstract}

Laser balloon ablation in patients with a left common pulmonary vein.

Shinichi Tachibana, M.D.; Kaoru Okishige, M.D., Ph.D., F.A.C.C., F.H.R.S.; Koji Sudo, M.D.; Takatoshi Shigeta, M.D.; Yuichiro Sagawa, M.D.; Manabu Kurabayashi, M.D., Ph.D.; Yasuteru Yamauchi, M.D., Ph.D.; *Masahiko Goya, M.D., Ph.D.; *Tetsuo Sasano, M.D., Ph.D.

Department of Cardiology, Japan Red Cross Yokohama City Bay Hospital

3-12-1 Shinyamashita, Nakaku, Yokohama, Kanagawa 231-8682, Japan

* Department of Cardiovascular Medicine, Tokyo Medical and Dental University (TMDU)

1-5-45 Yushima, Bunkyoku, Tokyo 113-8519, Japan

Correspondence to : Kaoru Okishige, M.D., Ph.D., F.A.C.C., F.H.R.S.

Japan Red Cross Yokohama City Bay Hospital

1-31-2 shinyamashita, Naka-ward, Yokohama, Kanagawa 231-0023, Japan 
Tel.: +81-45-628-6100 Fax: +81-45-622-8101

E-mail: okishige@yo.rim.or.jp

\section{Conflict of interest statement}

The authors declare no conflict of interest for this article.

\section{Data Availability}

Deidentified participant data will not be shared.

\section{Funding Sources}

The authors declare no conflict of interest for this article.

\section{Word count}

4973 words

\section{Abstract \\ Background:}

Pulmonary vein isolation (PVI) with a balloon-based visually guided laser ablation (VGLA) is regarded as a useful therapeutic tool for treating atrial fibrillation (AF). The clinical efficacy of a VGLA has never been fully investigated in patients with a left common pulmonary vein (LCPV).

\section{Objective:}

We investigated the procedural safety as well as clinical usefulness of VGLA in patients with an LCPV.

\section{Methods:}

This study consisted of 130 consecutive patients who underwent VGLA of de novo non-valvular paroxysmal $\mathrm{AF}$.

\section{Results:}

Eleven patients (8.5\%) had an LCPV (ostium maximal average diameter: $27.5 \pm 4.9 \mathrm{~mm}$, ostium minimal average diameter: $17.7 \pm 3.5 \mathrm{~mm})$. Nine out of $11(81.8 \%)$ LCPVs were successfully occluded and isolated at the ostium with a VGLA guided PVI. The ablation procedure time was significantly shorter in the patients with than without an LCPV $(61.5 \pm 15.4$ vs. $86.9 \pm 32.9 \mathrm{~min}, \mathrm{p}=0.01)$. There was no difference regarding the atrial tachyarrhythmia recurrence between those with and without an LCPV $(\mathrm{p}=0.18)$. A total of fifteen patients underwent a redo procedure, but reconnections were not observed in any of the LCPV patients.

\section{Conclusion:}

The VGLA guided PVI was a useful therapeutic tool even in patients with an LCPV. The presence of an LCPV was not associated with any atrial tachyarrhythmia recurrence.

\section{Keywords}

Left common pulmonary vein, catheter ablation, pulmonary vein isolation, laser balloon, atrial fibrillation

\section{Introduction}

Pulmonary vein isolation (PVI) is an effective treatment of atrial fibrillation (AF). A balloon-based visually guided laser ablation (VGLA) is considered to be a useful therapeutic tool for achieving a $\mathrm{PVI}_{1)}$. A prior multicenter study revealed that VGLA was non-inferior to radiofrequency ablation in terms of the efficacy and safety in curing paroxysmal and persistent $\mathrm{AF}_{2,3}$. Moreover, a recent prospective randomized study showed that VGLA was as effective and safe as a cryoballoon $(\mathrm{CB})$ guided $\mathrm{PVI}_{4}$ ). 
The shape of the pulmonary veins (PVs) and left atrium varies among the candidates for AF ablation, and a left common pulmonary vein (LCPV) is the most frequently observed anatomical abnormality that operators encounter in performing PVI. Although radiofrequency energy (RF) ablation can be applied in a point-by-point fashion at the LCPV ostium without any difficulty, the balloon may not be able to occlude the PVs, which is mandatory for a successful PVI. The results of a balloon-based PVI may not be satisfactory especially in patients with a relatively large LCPV, and we previously reported that a CB guided PVI was unsuccessful in $15 \%$ of patients with an $\mathrm{LCPV}_{5)}$. However, the clinical safety and efficacy of a VGLA has never been fully investigated in patients with an LCPV. We investigated the procedural safety and efficacy of VGLA in the patients with an LCPV.

\section{Methods}

\section{Study population}

Consecutive patients who underwent a PVI with VGLA as the first procedure for paroxysmal AF from July 2018 to January 2020 were eligible for this study. The subjects were patients who underwent catheter ablation of AF for standard clinical indications 1 ). Written informed consent for the ablation procedures was obtained from all patients. The definition of paroxysmal AF was according to the American Heart Association/American College of Cardiology/European Society of Cardiology guidelines, which is as follows: paroxysmal $\mathrm{AF}$ was defined as $\mathrm{AF}$ that self-terminated within 7 days 1 ). The study protocol was approved by the hospital's institutional human ethics committee. The study complied with the Declaration of Helsinki.

\section{Management before the catheter ablation}

A 12-lead electrocardiogram (ECG), chest X-ray, and echocardiogram were examined within 3 months before the catheter ablation. A transthoracic echocardiogram was performed to evaluate the left atrial diameter and left ventricular ejection fraction (LVEF). All patients underwent a 64-slice multi-detector row computed tomography (MDCT) within 1 week before the catheter ablation to obtain the configuration of the left atrial cavity and rule out any thrombi in the left atrium (LA) and left atrial appendage. A $60-100 \mathrm{ml}$ bolus of iodinated intravenous contrast was administered at a rate of $2.5-5 \mathrm{ml} / \mathrm{s}$, followed by a saline flush of 20-30 ml. The slice data of the MDCT image was reconstructed into a 3-dimensional volume rendering using computer software (ZAIO station2, ZAIOSOFT, Tokyo, Japan). The 3-dimensional image clarified the anatomy of the LA and PVs: left superior pulmonary vein (LSPV), left inferior pulmonary vein (LIPV), right inferior pulmonary vein (RSPV) and right inferior pulmonary vein (RIPV). An LCPV is generally defined as a common trunk length of $5 \mathrm{~mm}$ or more, short common trunk length of $5-15 \mathrm{~mm}$, and long common trunk length of more than $15 \mathrm{~mm}_{6,7)}$. In this study, an LCPV was defined as a PV with an entire length between the PV ostium and PV bifurcation of longer than $15 \mathrm{~mm}$ based on the MDCT image. All patients received anticoagulation therapy for at least 3 weeks before the ablation procedure. All antiarrhythmic drugs were discontinued for more than 5 half-lives before the ablation procedure, and amiodarone was not administered in any of the study patients.

\section{Catheter ablation procedure}

Catheter ablation was performed under general anesthesia using an intravenous administration of propofol and dexmedetomidine. A single-use supraglottic airway device (i-gel, NIHON KOHDEN, Tokyo, Japan) was inserted in all eligible patients, and an esophageal temperature thermocouple catheter (Esophaster, Japan Lifeline, Tokyo, Japan) was inserted through this airway device into the esophagus for continuous monitoring of the luminal esophageal temperature. Vascular access was acquired through the right internal jugular and femoral veins. A 20-pole catheter (BeeAT, Japan Lifeline, Tokyo, Japan) was inserted into the coronary sinus for electrogram recording, atrial pacing, and defibrillation. Two transseptal punctures were performed; one for a 12-Fr inner-diameter deflectable sheath (CardioFocus, MA, USA) and VGLA catheter and the other for a circular mapping catheter. Heparin was given as intravenous boluses followed by a constant infusion to maintain an activated clotting time of $>300$ seconds during the ablation procedure.

An EnSite NavX navigation system (St. Jude Medical, St. Paul, MN, USA) was used for 3-dimensional 
mapping. A mapping catheter (EPstar libero, Japan Lifeline, Tokyo, Japan) was used to construct the geometry of the LA and each PV. The bipolar electrogram filters were set between 30 and $500 \mathrm{~Hz}$.

The first generation VGLA catheter (HeartLight, CardioFocus, MA, USA) was inserted into the LA, and expanded trying to occlude each PVs. The laser balloon (LB) was inflated in a slow fashion to maximize the contact between the LB and cardiac tissue according to the size and shape of the target PV orifice. An LCPV isolation was performed with a maximal dilated LB (balloon size 9). If the LCPV could not be completely occluded with the maximal size of the LB, the LB was further inserted into the distal superior and inferior branches of the LCPV for the sake of isolating the distal branches the LCPV individually. The laser console uses aluminum gallium arsenide as a semiconductor laser element and emits a therapeutic laser with a wavelength of $980 \mathrm{~nm}$. The laser energy level was titrated according to the degree of tissue exposure between 5.5 and $12 \mathrm{~W}$ using the LightTrackTM software (CardioFocus, MA, USA). Every energy application was applied for 20 to 30 seconds depending on the applied laser energy. The area adjacent to the blood was applied with $5.5 \mathrm{~W}$ for 30 seconds in order to avoid an LB rupture. The laser energy was applied continuously overlapping each lesion by $50 \%$ around each PV ostium. The VGLA was prematurely terminated when the luminal esophageal temperature reached 39. The VGLA was performed in a sequence of the LSPV (or LCPV), LIPV, RSPV, and RIPV.

A 10-pole catheter was placed in the right subclavian vein or superior vena cava to pace the right phrenic nerve. The compound motor action potentials (CMAPs) were continuously measured, and the operators confirmed the tactile feedback of the diaphragmatic contractions during the VGLA of the RSPV or RIPV. The threshold for capturing the right-sided diaphragm was recorded, and phrenic nerve pacing was performed at a rate of 40 pulses per minute at an output exceeding the pacing threshold by $10 \% 8$ ). The VGLA was prematurely discontinued when the operator felt a reduction in the diaphragmatic contractions or the CMAP amplitude decreased by more than $30 \% 9$ ).

After the initial encirclement by the LB, the electrical isolation status of each PV was assessed. If all PVs were electrically isolated, dormant conduction was evaluated by an administration of adenosine triphosphate (ATP). The VGLA was subsequently applied at the electrical gap sites for touch-up ablation when the PVI was unsuccessful despite the initial encircling by the LB. When a PVI could not be successfully accomplished with only the VGLA, an irrigated RF catheter (TactiCath, Abbott, Illinois, USA) was used for touch-up ablation to eliminate all gap conduction sites. A successful PVI was defined as entrance and exit conduction block between the LA and PVs. After a successful initial PVI, it was reassessed 15 minutes after the last application. A voltage map of the PVs and LA was acquired before and after the PVI using a CARTO 3D mapping system (Figure 1 ).

A cavotricuspid isthmus ablation was also performed when atrial flutter was observed during the catheter ablation procedure or according to the discretion of the operator. No eligible patients underwent catheter ablation of non-PV foci. The RF energy was delivered with a maximum power of $35 \mathrm{~W}$, and the catheter tip temperature was limited to $42 \mathrm{degC}$. The ovality index was calculated according to the method of a previous report based on the CT image: $2 *$ (maximal diameter - minimal diameter) / (maximal diameter + minimal diameter) ${ }_{10)}$.

\section{Follow-up}

All patients were followed-up in the outpatient clinic at 1, 3, 6, 9, and 12 months after discharge. When the patients visited the outpatient clinic, a physical examination, 12-lead ECG, and review of the patients' symptoms were performed. All patients were encouraged to inform their physician of their physical status and whether they had any symptoms that were suggestive of an atrial tachyarrhythmia recurrence. If any atrial tachyarrhythmia recurrences were suspected, the cardiac rhythm was assessed using a 12-lead ECG. A Holter ECG examination was performed at 3 and 12 months after the VGLA procedure. Atrial tachyarrhythmia recurrence was defined as any sustained AF or atrial tachycardia (AT) lasting for i. 30 seconds, which appeared after the blanking period (i, 90 days after the catheter ablation).

\section{Statistical analysis}


Continuous variables are expressed as the mean $\pm \mathrm{SD}$, and the significant differences were analyzed with a Student's t test. Categorical data are expressed as the number and percentages, and were compared using an $\chi^{2}$ test or Fisher's exact test. Univariate and multivariate Cox proportional hazards regression analyses were performed on candidate variables to predict the dichotomous outcome. The variables with a $\mathrm{P}<$ 0.1 in the univariate analysis were input into the multivariate analysis. Survival curves were calculated using the Kaplan-Meier method and compared with the Log Rank test. A probability value of $<0.05$ was deemed significant. Statistical analyses were conducted using the EZR software (Saitama Medical Center, Jichi Medical University, Saitama, Japan), which is a graphical user interface for R (The R Foundation for Statistical Computing, Vienna, Austria).

\section{Results}

\section{Baseline characteristics}

Table 1 shows the baseline clinical characteristics of the present study patients. In this study, 130 patients (67\% male, age $66 \pm 12$ years) who underwent an initial PVI with VGLA were analyzed. The mean CHA2DS2VASc score was $2.0 \pm 1.5$ and mean LA diameter $38.4 \pm 6.1 \mathrm{~mm}$.

The RIPV could not be electrically isolated in 4 patients, because of prematurely terminating the VGLA due to the sudden failure of right phrenic nerve capture, which persisted until the end of the procedure. In all but those 4 patients, all PVs were successfully isolated. The LB ruptured in 3 patients during the procedure, and 26 patients underwent additional touch up RF ablation to achieve the PVI.

There were no significant differences in the age, gender, body mass index (BMI), or comorbidities such as hypertension, diabetes, and a history of congestive heart failure or a stroke between those with and without LCPV. The LA diameter and LVEF were also comparable. Moreover,Table 2 shows the procedural characteristics of the present study patients. The total procedure time and LB dwelling time in the LA were significantly shorter in the patients with an LCPV. There was no significant difference in the fluoroscopy time, number of ATP dormant conduction sites, and adjunctive RF touch-up ablation between the two groups. ATP dormant conduction was observed in 8 patients in the LSPV, 2 in the LIPV, 5 in the RSPV, 5 in the RIPV and none in the LCPV, respectively.

In the present study, 11 patients (8.5\%) had an LCPV (LCPV ostium maximal diameter: $27.5 \pm 4.9 \mathrm{~mm}$, LCPV ostium minimal diameter: $17.7 \pm 3.5 \mathrm{~mm}$, and LCPV ovality index: $0.44 \pm 0.15)$. The mean length between the LCPV ostium and bifurcation point of the superior and inferior PVs was $19.5 \pm 3.4 \mathrm{~mm}$. We could electrically isolate the LCPVs at the ostium with the VGLA in 9 patients, whereas the superior and inferior branches of the LCPV were isolated individually due to a large LCPV diameter in 2 patients. Those 2 patients had a significantly larger LCPV maximal ostium diameter than the remaining 9 patients $(34.8 \pm$ $1.1 \mathrm{~mm}$ vs. $25.9 \pm 3.8 \mathrm{~mm}, \mathrm{P}=0.01)$.

\section{Follow up}

There were no significant differences in the atrial tachyarrhythmia recurrence and rate of the administration of antiarrhythmic drugs during the blanking period of 90 days after the ablation procedure (Table 1 ). The mean follow-up period was $337 \pm 122$ (ranging from 90 to 580) days. After the blanking period of the catheter ablation, atrial tachyarrhythmias recurred in 20 patients $(15.4 \%$, AF recurrence in 19 patients including 3 with an LCPV, AT recurrence in 1 without an LCPV). A log-rank analysis demonstrated that there was no significant difference with regard to atrial tachyarrhythmia recurrence in the patients with and without an LCPV (Figure 2 ). In the univariate and multivariate analyses, recurrence during the blanking period after the PVI was an independent risk factor for an atrial tachyarrhythmia recurrence (Table 3 ).

Fifteen patients $(11.5 \%)$ underwent a second catheter ablation procedure including 3 (2.3\%) with an LCPV. Perimitral atrial flutter was observed in 1 patient without an LCPV. Reconnections of the RSPV, RIPV, LSPV, and LIPV were 8 out of 15 (53.3\%), 8 out of 15 (53.3\%), 4 out of $12(33.3 \%)$, and 4 out of $12(33.3 \%)$, respectively. The ATP dormant conduction sites were identical to the reconnected sites during the second 
ablation session in 2 patients. Of note, no reconnections were observed in any of the LCPV patients, and the LCPV ovality index was not associated with atrial tachyarrhythmia recurrence $(\mathrm{P}=0.63)$.

\section{Discussion}

One hundred and thirty patients who underwent VGLA using the first generation LB were analyzed in the present retrospective single center study. The main findings of our study were as follows: (1) we could successfully isolate the LCPVs at the ostium with only the VGLA in 9/11 patients $(81.8 \%$ ), (2) the total procedure time was significantly shorter in the patients with an LCPV than in those without $(174.9 \pm 31.6$ min vs. $148.2 \pm 19.9$ min, $\mathrm{P}<0.01$ ), and (3) the presence and configuration of the LCPV was not a significant predictor of any atrial tachyarrhythmia recurrence after the ablation procedure.

\section{Impact of an LCPV in balloon based PVI procedures}

There have been several prior reports on a CB guided PVI in patients of an LCPV. In the past, CB was considered technically challenging to achieve a PVI in patients with an LCPV, because the balloon size and lack of an appropriate balloon compliance made the complete occlusion of the PVs difficult. A previous study showed that atrial tachyarrhythmias were apt to recur after the ablation procedure using the first and second generation $\mathrm{CBs}_{5,11,12)}$. A point by point RF energy application may be suitable for the isolation of an LCPV, because it can isolate the PV at the LCPV ostium regardless of the size, length, or configuration of the LCPV. However, prior studies comparing the $2^{\text {nd }}$ generation $\mathrm{CB}$ and RF in patients with an LCPV reported no significant difference in the atrial tachyarrhythmia recurrence ${ }_{13,14)}$. Moreover, a recent randomized clinical trial (CIRCA-DOSE study) revealed the presence of an LCPV was associated with a trend towards higher rates of atrial tachyarrhythmia recurrence following the $\mathrm{PVI}_{15)}$. In this study, no significant difference in the atrial tachyarrhythmia recurrence was observed in the patients with an LCPV randomized to those undergoing a $\mathrm{CB}$ ablation and those undergoing an $\mathrm{RF}$ ablation $_{15)}$.

On the other hand, Nakamura et al. investigated the efficacy and long-term outcome of a Hot-balloon guided $\mathrm{PVI}_{16)}$. Of the patients in this study with an LCPV diameter of $<34 \mathrm{~mm}, 75 \%$ of the LCPVs were isolated at the ostium and $25 \%$ needed an individual isolation of the superior and inferior branches 16 ). Nakamura et al. revealed that the presence of an LCPV was not associated with recurrent AF, and significantly fewer Hot-balloon applications were sufficient for accomplishing the $\mathrm{PVI}_{16}$ ).

An LCPV is generally defined as having a length of $5 \mathrm{~mm}$ or more from the PV ostium to the bifurcation point (a short common trunk is $5-15 \mathrm{~mm}$ and a long common trunk more than $15 \mathrm{~mm}_{6,7}$ ), but we defined the LCPV length between the PV ostium and bifurcation point as longer than $15 \mathrm{~mm}$ on the MDCT findings. In the present study, a short LCPV was found in 21 patients. Since each short LCPV could not be isolated at the ostium, the superior and inferior branches of the LCPV had to be isolated individually. Therefore, those short LCPV cases were excluded from the present study analysis. There was no difference with regard to the total procedure time, ablation time for accomplishing the PVI, and atrial tachyarrhythmia recurrence between the patients with a short LCPV and those without an LCPV.

To the best of our knowledge, the present study was the first report to evaluate the efficacy of a VGLA guided PVI for an LCPV. The LB is so compliant that it can be inflated to any pressure and size change, which enables a maximum balloon/tissue contact regardless of the size or shape of each PV ostium. In the present study, we achieved a successful electrical isolation at the LCPV ostium in the majority of the patients with an LCPV. In addition, a comparable clinical result of atrial tachyarrhythmia recurrence could be observed in our study between the patients with and without an LCPV. Moreover, the ablation procedure time was shorter in the patients with an LCPV because the superior and inferior branches were isolated simultaneously with the VGLA as a result, which presumably attributed to a shorter catheter manipulation time. Although an electrical isolation at the LCPV ostium is difficult in cases with a large LCPV, VGLA could be selected as the first-line therapy in terms of the acute success and long-term clinical outcome.

\section{Long-term outcome and PVI durability}

The present study demonstrated that the predictors of any atrial tachyarrhythmia recurrence were not 
associated with the presence of an LCPV, but were related to the recurrence during the blanking period. In general, recurrence within 90 days of the blanking period is a major risk factor for any atrial tachyarrhythmia recurrence after the blanking period ${ }_{17-19}$, which was similar to the results of the VGLA in the present study.

VGLA enabled maintaining a high durable PVI. Nagase et al. and Okishige et al. demonstrated that the lesion depth, lesion volume, and maximum lesion diameter were associated with the laser output energy and total laser energy delivered in an in vitro model $_{20,21}$. A prior multicenter study revealed that $86 \%$ of the PVs remained isolated, and $62 \%$ of the patients had all their PVs remaining isolated after the VGLA guided $\mathrm{PVI}_{22}$. In the present study, PV reconnections were found in 24 out of $57(42.1 \%) \mathrm{PVs}$ among the patients who had recurrent atrial tachyarrhythmias and underwent a redo procedure. However, no electrical reconnections were found in any of the LCPV cases. The VGLA might create a highly durable lesion for LCPVs as derived from the present study results.

\section{Study limitations}

Our study had several limitations. First, this study was a single-center, retrospective analysis, and the study sample size was relatively small. A larger volume study might be needed to verify the present results. Second, the first generation VGLA (HeartLight, CardioFocus, MA, USA) was used in present study. The third generation VGLA (HeartLight X3, CardioFocus, MA, USA), which enables a single-sweep PVI with very short procedure times ${ }_{23}$, might be more effective for the LCPV isolation.

\section{Conclusion}

The clinical safety and long-term clinical efficacy of VGLA of paroxysmal AF was comparable between the patients between with and without an LCPV. A VGLA guided PVI could be regarded as a useful therapeutic tool even in patients with an LCPV.

\section{Acknowledgements}

There was no financial support associated with this study.

\section{Disclosures}

None.

\section{Reference}

1. Calkins H, Hindricks G, Cappato R, et al. 2017 HRS/EHRA/ECAS/APHRS/SOLAECE expert consensus statement on catheter and surgical ablation of atrial fibrillation. Heart Rhythm 2017;14:e275e444.

2. Dukkipati SR, Cuoco F, Kutinsky I, et al. Pulmonary vein isolation using the visually guided laser balloon: a prospective, multicenter, and randomized comparison to standard radiofrequency ablation. J Am Coll Cardiol 2015;66:1350-1360.

3. Schmidt B, Neuzil P, Luik A, et al. Laser Balloon or Wide-Area Circumferential Irrigated Radiofrequency Ablation for Persistent Atrial Fibrillation. Circ Arrhthm Electrophysiol 2017;10:e005767.

4. Chun JKR, Bordignon S, Last J, et al. Cryoballoon Versus Laserballoon: Insights From the First Prospective Randomized Balloon Trial in Catheter Ablation of Atrial Fibrillation. Circ Arrhythm Electrophysiol 2021;14:e009294.

5. Shigeta T, Okishige K, Yamauchi Y, et al. Clinical assessment of cryoballoon ablation in cases with atrial fibrillation and a left common pulmonary vein. J Cardiovasc Electrophysiol 2017;28:1021-1027.

6. McLellan AJ, Ling LH, Ruggiero D, et al. Pulmonary vein isolation: the impact of pulmonary venous anatomy on long-term outcome of catheter ablation for paroxysmal atrial fibrillation. Heart Rhythm 2014;11:549-556.

7. Cabrera JA, Ho SY, Climent V, Sanchez-Quintana D. The architecture of the left lateral atrial wall: a particular anatomic region with implications for ablation of atrial fibrillation. Eur Heart J 2008;29:356362. 
8. Okishige K, Aoyagi H, Kawaguchi N, et al. Novel method for earlier detection of phrenic nerve injury during cryoballoon applications for electrical isolation of pulmonary veins in patients with atrial fibrillation. Heart Rhythm 2016;13:1810-1816.

9. Mondesert B, Andrade JG, Khairy P, et al. Clinical experience with a novel electromyographic approach to preventing phrenic nerve injury during cryoballoon ablation in atrial fibrillation. Circ Arrhythm Electrophysiol 2014;7:605-611.

10. Schmidt M, Dorwarth U, Straube F, et al. Cryoballoon in AF ablation: impact of PV ovality on AF recurrence. Int J Cardiol 2013;167:114-120.

11. Kubala M, Hermida JS, Nadji G, Quenum S, Traulle S, Jarry G. Normal pulmonary veins anatomy is associated with better AF-free survival after cryoablation as compared to atypical anatomy with common left pulmonary vein. Pacing Clin Electrophysiol 2011;34:837-843.

12. Beiert T, Lodde PC, Linneborn LPT, et al. Outcome in patients with left common pulmonary vein after cryoablation with second-generation cryoballoon. Pacing Clin Electrophysiol 2018;41:22-27.

13. Yamaguchi M, Miyazaki S, Kajiyama T, et al. Pulmonary vein isolation in patients with a left common pulmonary vein: comparison between second-generation cryoballoon and radiofrequency ablation. J Cardiol 2019;73:292-298.

14. Coutino HE, Stroker E, Takarada K, et al. Radiofrequency versus cryoballoon ablation for atrial fibrillation in the setting of left common pulmonary veins. Pacing Clin Electrophysiol 2019;42:14561462.

15. Larsen JM, Deyell MW, Macle L, et al. Impact of Left Common Pulmonary Veins in the ContactForce vs. Cryoballoon Atrial Fibrillation Ablation (CIRCA-DOSE) Study. J Cardiovasc Electrophysiol 2020;31:2300-2307.

16. Nakamura Y, Sohara H, Ihara M. Acute efficacy and clinical outcomes using HotBalloon for pulmonary vein isolation in patients with a left common pulmonary vein. J Cardiovasc Electrophysiol 2019;30:12411249 .

17. Andrade JG, Macle L, Khairy P, et al. Incidence and significance of early recurrences associated with different ablation strategies for AF: a STAR-AF substudy. J Cardiovasc Electrophysiol 2012;23:12951301.

18. Nalliah CJ, Lim TW, Kizana E, et al. Clinical significance of early atrial arrhythmia type and timing after single ring isolation of the pulmonary veins. Europace 2015;17:1038-1044.

19. Themistoclakis S, Schweikert RA, Saliba WI, et al. Clinical predictors and relationship between early and late atrial tachyarrhythmias after pulmonary vein antrum isolation. Heart Rhythm 2008;5:679-685.

20. Nagase T, Asano S, Yukino M, et al. Influence of various energy settings and overlap ratios on size and continuity of lesions in a laser balloon ablation in vitro model. J Cardiovasc Electrophysiol 2019;30:1330-1338.

21. Okishige K, Shigeta T, Nakamura RA, et al. Experimental Study With Regard to the Effects of Energy Titration of the Laserballoon on the Lesion Creation Using Porcine Myocardium. Int Heart $\mathrm{J}$ 2020;61:121-127.

22. Dukkipati SR, Neuzil P, Kautzner J, et al. The durability of pulmonary vein isolation using the visually guided laser balloon catheter: multicenter results of pulmonary vein remapping studies. Heart Rhythm 2012;9:919-925.

23. Heeger $\mathrm{CH}$, Tiemeyer CM, Phan HL, et al. Rapid pulmonary vein isolation utilizing the thirdgeneration laserballoon - The PhoeniX registry. Int J Cardiol Heart Vasc 2020;29:100576.

Table 1 . Patient characteristics

\begin{tabular}{llll}
\hline Variable & Overall $(\mathrm{n}=130)$ & $\mathrm{LCPV}-(\mathrm{n}=119)$ & $\mathrm{LCPV}+(\mathrm{n}=11)$ \\
\hline Age, years & $66.0 \pm 11.8$ & $65.7 \pm 11.8$ & $69.0 \pm 12.1$ \\
Female, $\mathrm{n}$ & $43(33.1 \%)$ & $39(32.8 \%)$ & $4(36.3 \%)$ \\
$\mathrm{BMI}, \mathrm{kg} / \mathrm{m}^{2}$ & $23.8 \pm 3.7$ & $23.8 \pm 3.8$ & $24.6 \pm 3.0$ \\
Hypertension, $\mathrm{n}$ & $54(41.5 \%)$ & $48(40.3 \%)$ & $6(54.5 \%)$ \\
Diabetes, $\mathrm{n}$ & $10(7.7 \%)$ & $8(6.7 \%)$ & $2(18.2 \%)$
\end{tabular}




\begin{tabular}{llll}
\hline Variable & Overall $(\mathrm{n}=130)$ & $\mathrm{LCPV}-(\mathrm{n}=119)$ & $\mathrm{LCPV}+(\mathrm{n}=11)$ \\
\hline Heart failure, $\mathrm{n}$ & $8(6.2 \%)$ & $8(6.7 \%)$ & $0(0 \%)$ \\
Stroke or TIA, $\mathrm{n}$ & $11(8.5 \%)$ & $10(8.4 \%)$ & $1(9.0 \%)$ \\
$\mathrm{CHA}_{2} \mathrm{DS}_{2}$ VASc score & $2.0 \pm 1.5$ & $2.0 \pm 1.4$ & $2.3 \pm 1.9$ \\
$\mathrm{LA} \mathrm{diameter}, \mathrm{mm}$ & $38.4 \pm 6.1$ & $38.4 \pm 6.1$ & $38.2 \pm 5.5$ \\
$\mathrm{LVEF}, \%$ & $65.9 \pm 9.0$ & $65.8 \pm 9.1$ & $66.1 \pm 8.1$ \\
Atrial tachyarrhythmia recurrence during the blanking period, $\mathrm{n}$ & $22(16.9 \%)$ & $20(16.8 \%)$ & $2(18.2 \%)$ \\
AAD the blanking period, $\mathrm{n}$ & $8(6.2 \%)$ & $8(6.7 \%)$ & $0(0 \%)$ \\
\hline
\end{tabular}

$\mathrm{LCPV}=$ left common pulmonary vein; $\mathrm{BMI}=$ body mass index; TIA $=$ transient ischemic attack; LA $=$ left atrium; $\mathrm{LVEF}=$ left ventricular ejection fraction; $\mathrm{AAD}=$ antiarrhythmic drug.

Values are expressed as the mean $\pm \mathrm{SD}$ or as $\mathrm{n}(\%) .{ }^{*} \mathrm{P}$ value is compared between the LCPV - and LCPV + groups, respectively.

Table 2 . Procedural characteristics of the patients with and without an LCPV

\begin{tabular}{lllll}
\hline Procedural valuables & Overall $(\mathrm{n}=130)$ & LCPV $-(\mathrm{n}=119)$ & LCPV $+(\mathrm{n}=11)$ & *P value \\
\hline Total procedure time, min & $172.7 \pm 31.7$ & $174.9 \pm 31.6$ & $148.2 \pm 19.9$ & $<0.01$ \\
LB dwelling time in LA, min & $84.8 \pm 32.5$ & $86.9 \pm 32.9$ & $61.5 \pm 15.4$ & 0.01 \\
Fluoroscopy time, min & $55.5 \pm 15.3$ & $55.8 \pm 15.3$ & $51.9 \pm 15.7$ & 0.44 \\
Laser mean output, W & & & & \\
RSPV & $8.7 \pm 1.4$ & $8.7 \pm 1.5$ & $9.3 \pm 1.2$ & 0.18 \\
RIPV & $7.9 \pm 1.4$ & $7.9 \pm 1.4$ & $8.0 \pm 1.5$ & 0.82 \\
LSPV & $8.6 \pm 1.5$ & $8.6 \pm 1.5$ & & \\
LIPV & $7.7 \pm 1.6$ & $7.7 \pm 1.6$ & & \\
LCPV & $8.5 \pm 1.3$ & & $8.5 \pm 1.3$ & \\
Total energy, J & & & \\
RSPV & $6014.9 \pm 2667.3$ & $6037.1 \pm 2741.3$ & $5774.6 \pm 1742.0$ & 0.76 \\
RIPV & $4489.5 \pm 1506.7$ & $4507.1 \pm 1522.3$ & $4290.9 \pm 1374.6$ & 0.67 \\
LSPV & $6745.3 \pm 3070.0$ & $6745.3 \pm 3070.0$ & & \\
LIPV & $4647.9 \pm 2374.6$ & $4647.9 \pm 2374.6$ & & \\
LCPV & $7610.8 \pm 4681.5$ & & $7610.8 \pm 4681.5$ & \\
ATP dormant conduction, $\mathrm{n}$ & $19(14.6 \%)$ & $18(15.1 \%)$ & $1(9.0 \%)$ & 1.0 \\
Radiofrequency touch up, $\mathrm{n}$ & $26(20.0 \%)$ & $23(19.3 \%)$ & $3(27.2 \%)$ & 0.46 \\
CTI ablation, n & $119(91.5 \%)$ & $109(91.6 \%)$ & $10(90.9 \%)$ & 1.0 \\
\hline
\end{tabular}

$\mathrm{LCPV}=$ left common pulmonary vein; $\mathrm{LB}=$ laser balloon; LA $=$ left atrium; $\mathrm{RSPV}=$ right superior pulmonary vein; RIPV = right inferior pulmonary vein; $L S P V=$ left superior pulmonary vein; LIPV = left inferior pulmonary vein; ATP $=$ adenosine triphosphate; CTI $=$ cavotricuspid isthmus.

Values are expressed as the mean $\pm \mathrm{SD}$ or as $\mathrm{n}(\%) .{ }^{*} \mathrm{P}$ value is compared between the LCPV - and LCPV + groups, respectively.

Table 3. Univariate and multivariable analysis for the prediction of an atrial tachyarrhythmia recurrence

\begin{tabular}{lllll}
\hline & Univariate analysis & Univariate analysis & Multivariate analysis & Multivariate an \\
\hline & HR $(95 \% \mathrm{CI})$ & $\mathrm{P}$ value & HR $(95 \% \mathrm{CI})$ & $\mathrm{P}$ value \\
Age & $1.03(0.98-1.08)$ & 0.20 & & \\
Female & $1.16(0.46-2.96)$ & 0.75 & &
\end{tabular}




\begin{tabular}{lllll}
\hline & Univariate analysis & Univariate analysis & Multivariate analysis & Multivariate an \\
\hline $\mathrm{BMI}_{\mathrm{CHA}} \mathrm{DS}_{2}$ VASc score & $1.0(0.89-1.12)$ & 0.96 & & \\
LA diameter & $1.28(0.95-1.72)$ & 0.10 & $1.28(0.95-1.73)$ & 0.11 \\
LCPV & $1.05(0.98-1.13)$ & 0.18 & & \\
Recurrence during the blanking period & $2.42(0.70-8.40)$ & 0.16 & $3.04(1.19-7.73)$ & 0.02 \\
\hline
\end{tabular}

$\mathrm{BMI}=$ body mass index; LA = left atrium; LCPV = left common pulmonary vein; HR = hazard ratio; CI $=$ confidence interval.

\section{Figure Legends}

Figure 1. An example of a voltage map of the left atrium and pulmonary veins before and after the PVI (purple area; a voltage of more than $0.5 \mathrm{mV}$, gray area; a voltage of less than $0.1 \mathrm{mV}$ ). The distance between the LCPV ostium and bifurcation was $18 \mathrm{~mm}$. The LCPV was successfully isolated with an LB inserted into the LCPV superior branch.

$\mathrm{PVI}=$ pulmonary vein isolation; $\mathrm{LCPV}=$ left common pulmonary vein; $\mathrm{LB}=$ laser balloon .

Figure 2. Long-term freedom from atrial tachyarrhythmia recurrence after an initial VGLA guided PVI.

VGLA $=$ visually guided laser ablation; PVI = pulmonary vein isolation.

\section{Figures}

\section{Figure 1.}

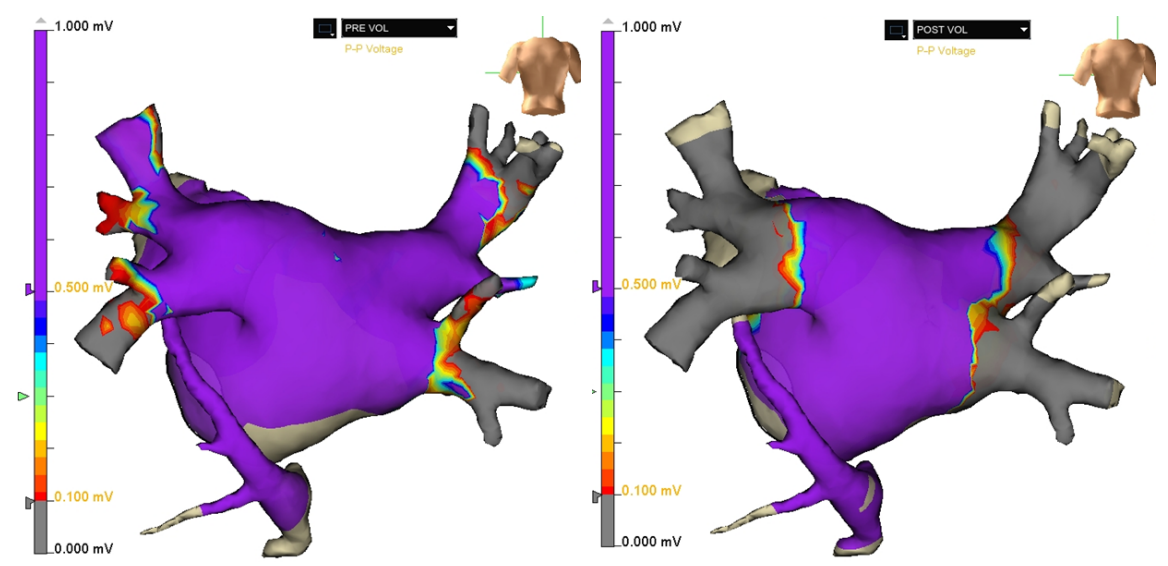

Figure 2. 


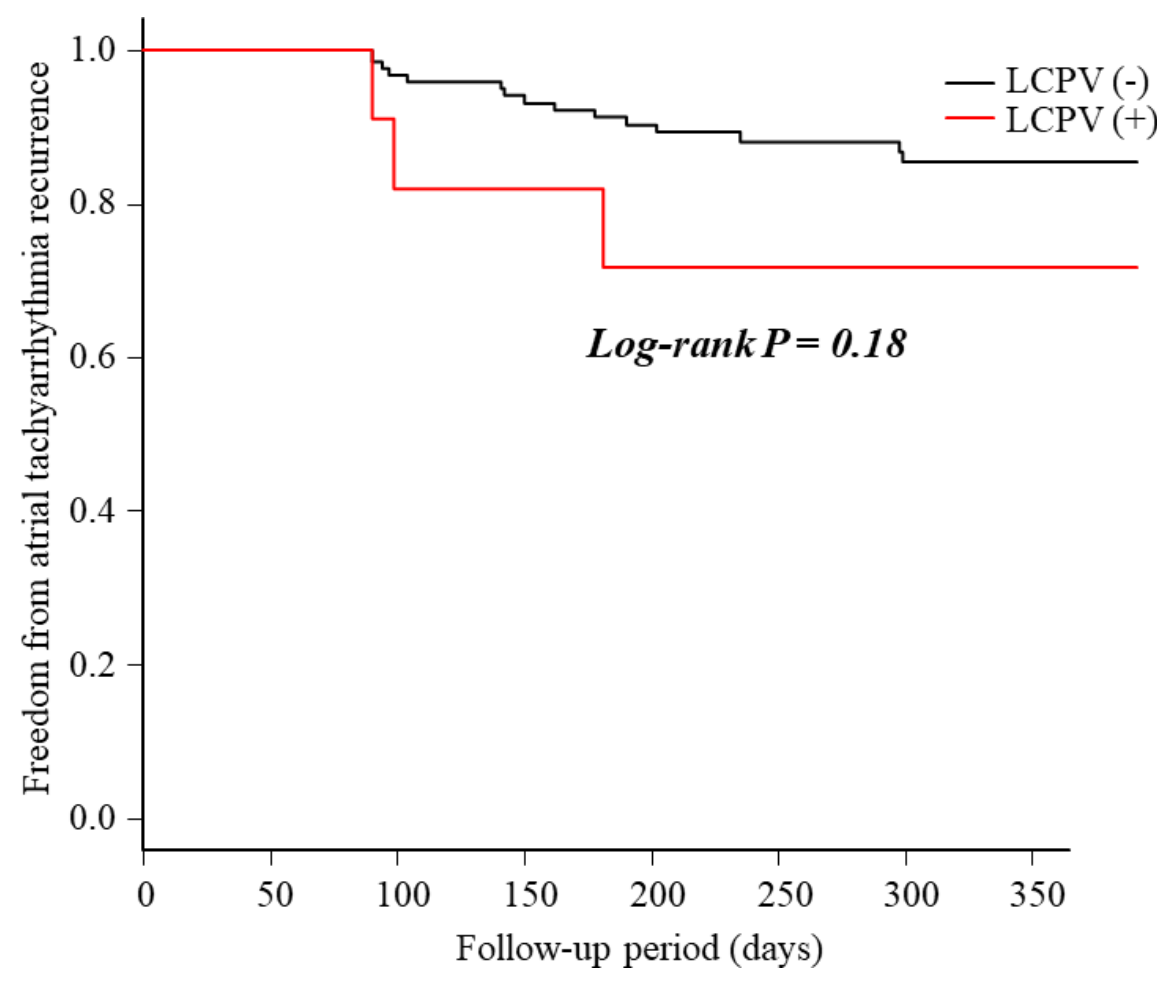

